\title{
Gene Profiles in a Smoke-Induced COPD Mouse Lung Model Following Treatment with Mesenchymal Stem Cells
}

\author{
You-Sun Kim ${ }^{1,2}$, Nurdan Kokturk ${ }^{3}$, Ji-Young Kim², Sei Won Lee ${ }^{1,4}$, Jaeyun Lim', Soo Jin Choi ${ }^{5}$, Wonil Oh${ }^{5}$, \\ and Yeon-Mok $\mathrm{Oh}^{1,4, *}$
}

\begin{abstract}
Mesenchymal stem cells (MSCs) effectively reduce airway inflammation and regenerate the alveolus in cigarette- and elastase-induced chronic obstructive pulmonary disease (COPD) animal models. The effects of stem cells are thought to be paracrine and immune-modulatory because very few stem cells remain in the lung one day after their systemic injection, which has been demonstrated previously. In this report, we analyzed the gene expression profiles to compare mouse lungs with chronic exposure to cigarette smoke with non-exposed lungs. Gene expression profiling was also conducted in a mouse lung tissue with chronic exposure to cigarette smoke following the systemic injection of human cord blood-derived mesenchymal stem cells (hCB-MSCs). Globally, 834 genes were differentially expressed after systemic injection of hCB-MSCs. Seven and 21 genes, respectively, were up-and downregulated on days 1,4 , and 14 after HCB-MSC injection. The $\mathrm{Hbb}$ and $\mathrm{Hba}$, genes with oxygen transport and antioxidant functions, were increased on days 1 and 14 . A serine protease inhibitor was also increased at a similar time point after injection of hCB-MSCs. Gene Ontology analysis indicated that the levels of genes related to immune responses, metabolic processes, and blood vessel development were altered, indicating host responses after hCB-MSC injection. These gene expression changes suggest that MSCs induce a regeneration mechanism against COPD induced by cigarette smoke. These analyses provide basic data for understanding the regeneration mechanisms promoted by hCB-MSCs in cigarette smokeinduced COPD.
\end{abstract}

\footnotetext{
${ }^{1}$ University of Ulsan College of Medicine, ${ }^{2}$ Asan Institute for Life Sciences, Seoul 05505, Korea, ${ }^{3}$ Department of Pulmonology, Gazi University, Ankara, Turkey, ${ }^{4}$ Department of Pulmonary and Critical Care Medicine, Asan Medical Center, Seoul 05505, Korea, ${ }^{5}$ Biomedical Research Institute, MEDIPOST Co., Ltd., Seoul, Korea

*Correspondence: ymoh55@amc.seoul.kr
}

Received 11 April, 2016; revised 7 September, 2016; accepted 7 September, 2016; published online 31 October, 2016

Keywords: gene profile, human cord blood-derived mesenchymal stem cells, microarray, smoke-induced chronic obstructive pulmonary disease

\section{INTRODUCTION}

Chronic obstructive pulmonary disease (COPD) is characterized by airway inflammation and alveolar wall destruction (emphysema) that result from exposure to cigarette smoke (Rabe et al., 2007). The developmental mechanism of COPD has been suggested to involve cigarette smoke-induced aimay inflammation, oxidative stress, and alveolar destruction from an imbalance between proteases and anti-proteases (MacNee and Tuder, 2009; Yoshida and Tuder, 2007). Although current treatments are available to inhibit airway inflammation and relax contracted airways, there is no therapy that regenerates destroyed alveoli (Aaron et al., 2007; Calverley et al., 2007). Thus, recent studies to improve COPD treatment have focused on the control of anti-oxidative and anti-protease activities.

Cell therapy using various stem cells has shown beneficial therapeutic effects in animal models of COPD (Huh et al., 2011; Katsha et al., 2011; Schweitzer et al., 2011). Our recent studies showed that MSCs from the bone marrow and cord blood successfully improved the emphysema component of COPD (Huh et al., 2011). Recently, we reported that most stem cells injected systemically disappeared within one day, when the stem cells were tracked by fluorescent labeling or with human-based alu sequence quantification (Kim et al., 2014). The early disappearance of infused stem cells has also been observed in other studies (Lee et al., 2009). Most stem cell biologists agree that stem cells can differentiate into bone, adipose tissue, and cartilage in vitro, but exert their therapeutic effects via paracrine and immunomodulatory functions in vivo (Murphy et al., 2013).Stem cells may deliver some signals to host cells, inducing a regenerative mechanism against alveolar destruction in the COPD lung. Therefore, studies focused on changes in gene expression profiles in host cells may be important for understanding MSCs regeneration mechanisms in destroyed alveoli.

Previous studies have analyzed gene profiles in the presence and absence of cigarette exposure. However, no studies have examined the changes in gene profiles following MSC injection in a cigarette smoke-induced COPD model. Therefore, the goal of the current study was to analyze the changes in gene expression profiles in the cigarette smoke-induced COPD mouse lung following systemic injection of human cord bloodderived (hCB)-MSCs over time on days 1, 4, and 14. Using a mouse WG-6 expression bead array based on Illumina, we 


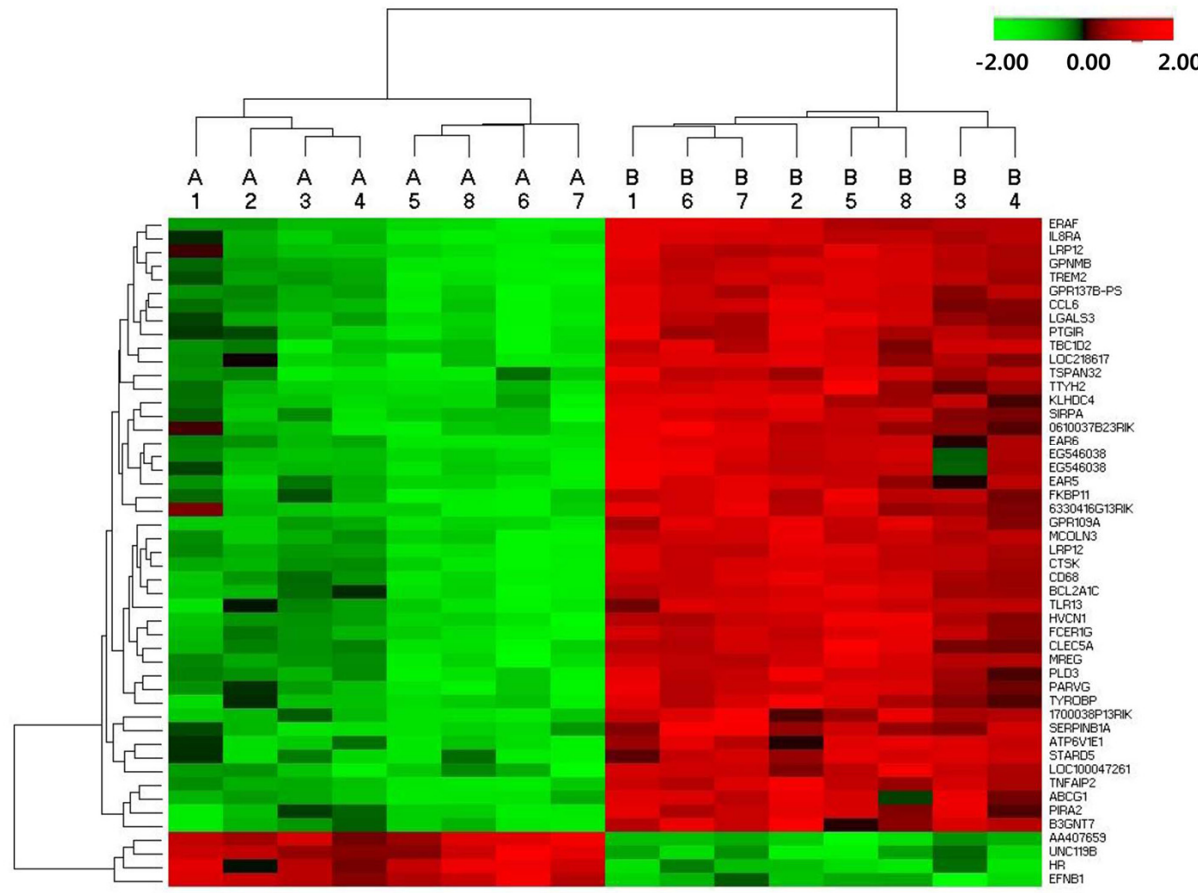

Fig. 1. Heat map of DEG with $p$ value $<0.01$. (A) and $(B)$ indicates normal air and cigarette exposure, respectively ( $n=8$, each group). Each gene is represented on the right side of the heat map.

compared gene expression profiles following cigarette exposure and non-exposure. Next, we compared the gene expression profiles of two groups, an hCB-MSC-injected group and a non-injected group, both of which were induced to have COPD lungs by a 6-month exposure to cigarette smoke before systemic injection of hCB-MSCs.

\section{MATERIALS AND METHODS}

\section{Animal experiments}

C57BL/6J mice were purchased from OrientBio (Korea). Mice were bred in specific pathogen-free facilities at the Asan Medical Center. All live mouse experiments were approved by the Institutional Animal Care and Use Committee of the Asan Medical Center (Korea). Seven-week-old female C57BL/6J mice were exposed to commercial cigarette smoke (Eighty Eight Lights, KT\&G, Daejeon, Korea) 5 days per week for 6 months as we described previously(Huh et al., 2011). Briefly, 30-40 mice were settled in an inhalation box $(50 \times 40 \times 30 \mathrm{~cm})$ connected to a pump, and exposed to 12 cigarettes. Control mice were bred in cages with clean room air. Human cord blood MSCs were provided by MEDIPOST Co., Ltd (Korea). hCBMSCs were cultured with alpha-minimum essential medium (Gibco Life Technologies, USA) and subcultured using $0.25 \%$ trypsin-EDTA (Gibco Life Technologies). Mice exposed to 6 months of cigarette smoke were intravenously injected with $5 \times$ $10^{4} \mathrm{hCB}-\mathrm{MSCs}$. Mice were then sacrificed and their lungs were collected on days 1, 4, and 14 following injection of hCB-MSCs. RNA later was used to maintain RNA stability during mouse lung collection.

\section{RNA isolation}

Total mRNA from lung tissue was prepared using Trizol (Invitrogen Life Technologies, USA) and purified using RNeasy columns (Qiagen, Germany) according to the manufacturer's protocol. The purity and quantity of the isolated RNA were evaluated using aND-1000 spectrophotometer (NanoDrop Technologies Inc., USA), and a quality check of the RNA was performed using a 2100 Bioanalyzer (Agilent Technologies, USA). Only RNA samples with an RNA integrity number $>7.5$ were used for the microarray experiment.

\section{Microarray experiments}

RNA was amplified and purified using the Ambionlllumina RNA amplification kit (Ambion, USA) according to the manufacturer's instructions. Biotinylated cRNA $(1.5 \mu \mathrm{g})$ was hybridized to each MouseWG-6 expression bead array (Illumina, Inc., USA), and the array signal was detected using Amersham fluorolink streptavidin-Cy3 (GE Healthcare Biosciences, UK). Arrays were scanned with an Illumina bead array reader confocal scanner.

\section{Immunohistochemistry}

The lung was inflated with $0.5 \%$ low-melting agarose and fixed using $4 \%$ formalin, and then embedded in paraffin and cut into 4- $\mu \mathrm{m}$-thick sections for immunohistochemistry. The sections were stained using an Immunohistochemistry accessory kit (Bethyl Laboratories, Inc., USA) with anti-Egr-1 antibody (1:250, Abcam, UK) and then observed by microscopy.

\section{Data analysis}

All data analysis of differentially expressed genes was conducted using R 2.15.1. Raw expression data was log2-transformed and normalized by a quantile method. Statistical significance of the expression data was determined using a $t$-test, and we restricted our interest to the gene list with $p$-values compared to cigarette exposure or non-exposure. The genes analyzed included those show a 1.3-foldchange between the smokeexposed group and smoke + hCB-MSCs injected group at each 
A

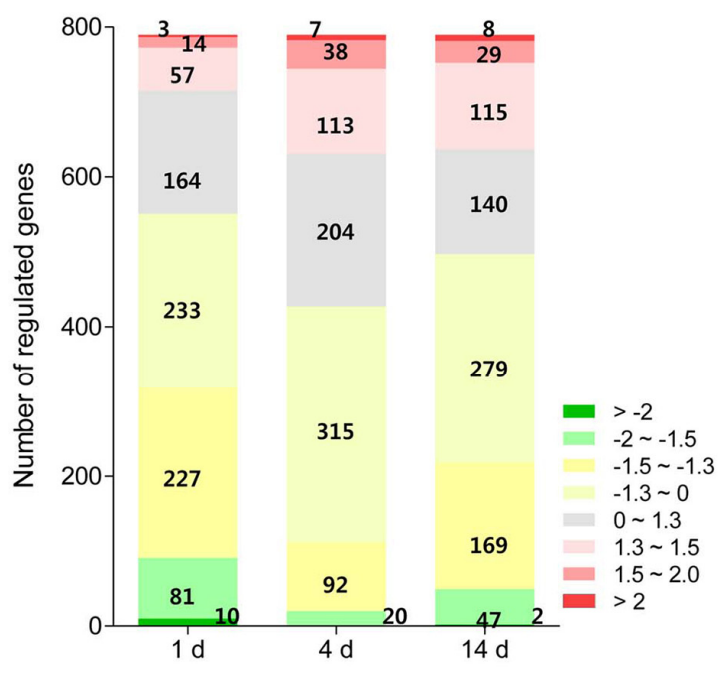

Time (day) after systemic injected MSCs
B

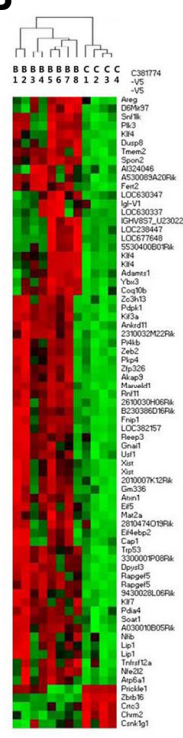

C

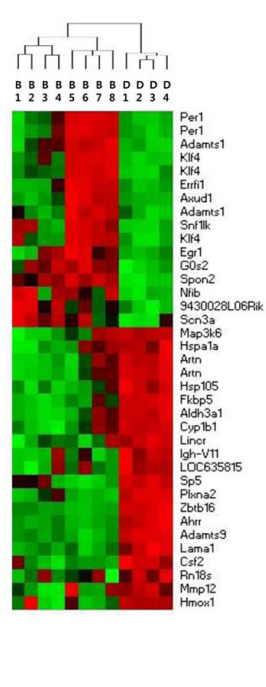

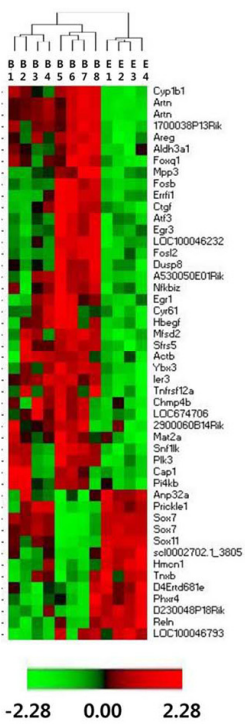

Fig. 2. Gene regulation overtime. The numbers and heat map of regulated genes were divided according to fold-change and up-or downregulation.

A

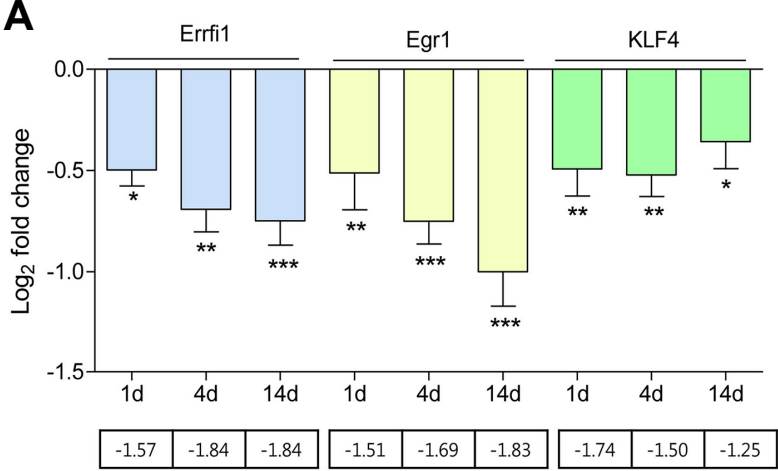

B

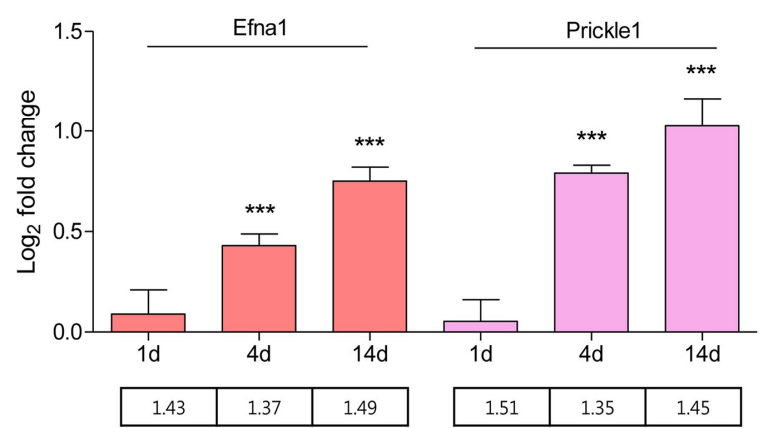

Fig. 3. Validation of microarray using quantitative PCR (qPCR). qPCR was performed with selected 5 genes:Effri1, Egf1, KLF4, Efna1, and Prickle1. All qPCR steps were performed as technical duplicates and the expression levels of selected genes were normalized to that of $\beta$-actin. ${ }^{*} p<0.05,{ }^{* \star} p<0.01$, ${ }^{\star \star *} p<0.001 \mathrm{com}$ pared to MSC non-injection group using $t$-test. The boxes below the each graph are represented as microarray fold-change results ( $t$ test, $p<0.05)$. time point. After statistical analysis, we assigned GO terms to the genes with altered expression using DAVID (http://david. abcc.ncifcrf.gov/home.jsp).

\section{RESULTS}

Gene expression profiles following chronic exposure to cigarette smoke

A $t$-test was performed to identify differentially expressed genes (DEGs) based on their p-values. Forty-nine and 231DEGs had $p$-values of $<0.01$ and $<0.05$, respectively. Figure 1 shows the heat map indicating that 45 genes were up-regulated and 4 genes were down-regulated in the cigarette-exposed group compared to the non-exposed group. The top 10 genes are listed in Table 1 and 231 of DEGs were subjected to GO evaluation using DAVID. Immune response, inflammatory response, and apoptosis (cell death) were enriched in up-regulated DEGs. Down-regulated DEGs were enriched in cell and biological adhesion (Table 2).

Gene expression profiles following injection of hCB-MSCs A $t$-test was performed to select genes that were significantly up- or down-regulated following injection of hCB-MSCs. We identified genes showing a $>1$.3-foldchange that were significantly different between the hCB-MSC-injected group and noninjected group. A total of 834 genes were differently expressed between the two groups; 392, 270, and 368 showed significant changes in expression at 1, 4, and 14 days after injection of hCB-MSCs, respectively. Figure 2 shows the summary and heat map of the $t$-test and the number of up- and downregulated genes in the 1.3-foldchange group. Some representative genes were subjected to quantitative PCR (qPCR) for technical validation and most microarray data matched with the qPCR results (Fig. 3 ).

Figure 4 shows the genes specifically altered by injection of hCB-MSCs at each time point and in common between the 
A

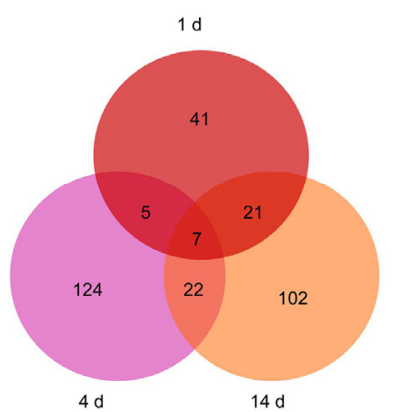

B Down-regulated genes

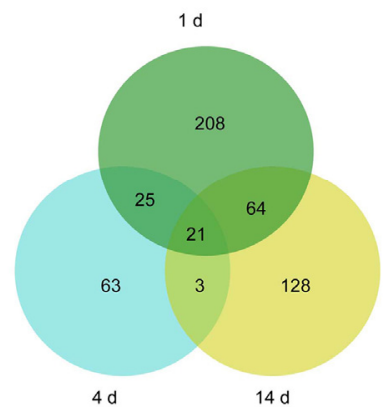

Fig. 4. Venn diagram of regulated genes for the three sampling times. (A) Up-regulated genes. (B) Down-regulated genes.

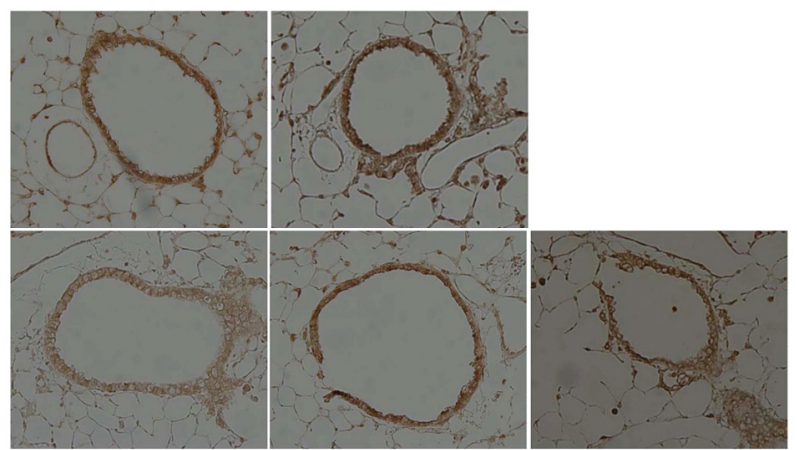

Fig. 5. Immunohistochemistry for Egr-1 in lungs. Upper left: normal air exposure, upper right: cigarette exposure, lower left, middle, right: 1,4 , and 14 days after MSC injection during cigarette exposure, respectively

different time points using a Venn diagram. Seven genes and 21 genes were continuously up- and down-regulated, respectively, following injection of hCB-MSCs. The common up- and down-regulated genes are shown in Table 2. The number of up-regulated genes was the lowest at 1 day after injection of hCB-MSCs compared to the other time points and increased in a time-dependent manner. In contrast, the largest number of down-regulated genes showing differential expression was observed on day 1 after injection of hCB-MSCs. Figure 5 was shows the immunohistochemistry results for Egr-1 in the lungs. The Egr-1 gene was down-regulated in both groups following hCB-MSC injection. The results revealed only a minimal correlation between mRNA expression and protein expression.

The DEGs between the hCB-MSCs-injected and noninjected groups were categorized based on time and direction of expression. We also performed GO analysis using DAVID. The top 20 up-regulated genes at each time point are listed in Table 3. We also represent the fold-change following cigarette exposure in Table 3. The expression of oxygen transport- and anti-oxidant function-related genes such as $H b b$ and $H b a$ were increased on days 1 and 14. One serine protease inhibitor (Serpina) was increased at a nearly time point after MSC injection. Ahrr, which is involved in reducing oxidative metabolism, increased on day 4 . In a previous report, microarray was performed to evaluate COPD and healthy subjects and GO term enrichment analysis was conducted for DEGs. They identified
COPD-related GO terms such as immune response, inflammatory response, wounding, chemotaxis, and cell adhesion (Yang et al., 2013). Our results also generated these COPD-related GO terms (Table 4). The top 20 up-and down-regulated genes over time are listed in Supplementary Table 2 and 3. Cyp1b1, a representative gene up-regulated by smoke exposure in mice, was decreased by MSC injection on day 14. Hematopoiesis-, immune response-, and cholesterol-related genes were upregulated on day 1 . Down-regulated genes on day 1 were related to transcription, metabolic process, and blood/vasculature development (Supplementary Table 4). Supplementary Table 5 shows the results of GO term analysis for up- and downregulated genes on day 4 . Up-regulated genes on day 4 were related to wound healing, immune responses, and blood circulation. Blood and tube development-, transcription-, and metabolic process-related genes were down-regulated on day 4 . The results of GO term analysis for up-and down-regulated genes on day 14 are shown in Supplementary Table 6. In particular, the GO term categories of blood vessel and vasculature development appeared in both up- and down-regulated genes.

\section{DISCUSSION}

In the present study, we analyzed the gene expression profiles following cigarette exposure or non-exposure as well as hCBMSC-injected or non-injected mice. We focused on the changes in gene profiles between hCB-MSCs-injected vs. noninjected mouse lungs following the induction of COPD by chronic exposure to cigarette smoke and found that gene expression profiles varied with after injection of hCB-MSCs. Genes involved in oxidative stress, xenobiotic metabolic processes, and anti-protease functions were detected most frequently. In addition, GO term analysis showed that genes related to immune responses, blood vessel development, and metabolic processes were differentially expressed on days 1 and 4 after hCB-MSC injection compared to controls. DEGs on day 14 after injection of hCB-MSCs were related to cell growth regulation. Our results suggest that immune responses, oxidative stress, and transcription were regulated at a nearly stage after hCB-MSC injection (on days 1 and 4), and that blood vessel development and cell growth regulation predominated at a later stage after hCB-MSC injection (on day 14).

In previous reports, MSCs showed regenerative properties in animal models with smoke- and elastase-induced COPD (Huh et al., 2011; Katsha et al., 2011; Schweitzer et al., 2011). Recently, we reported that systemically injected MSCs were observed for only 1 day after cell injection in an elastase-induced emphysema model (Kim et al., 2014). Other reports failed to detect injected MSCs; therefore, stem cell biologists have attempted to explain the therapeutic effect of mesenchymal stem cells in these models in the context of the regulation of host cells or host microenvironment (Henning et al., 2014; Liang et al., 2014; Suga et al., 2014). To understand the detailed mechanisms of stem cell therapy, understanding the changes in host genes following MSC injection is required. Thus, we focused on the host changes based on the profiles of DEGs in this study.

Previous studies analyzed the gene expression profiles in the lungs of smoke-exposed mice (Halappanavar et al., 2009; Rangasamy et al., 2009).Inflammatory signaling was upregulated following cigarette exposure. We also detected upregulation of inflammatory-related genes such as GPR109A (HCA2), TNFAIP2, IL-8RA, and CCL6 (Table 1) (Chen et al., 2008; Rangasamy et al., 2009; Zandi-Nejad et al., 2013). A previous report showed that most genes altered by exposure to 
smoke returned to a basal level of expression 6 weeks after smoking cessation (Rangasamy et al., 2009). Temporal alterations in the gene expression profile during the progression of emphysema have also been analyzed in the lungs of mice exposed to smoke for 5 h, 8 days, 1.5 months, and 6 months. Genes up-regulated by smoke exposure are categorized as phase I genes such as Cyp1a1 and Cyp1b1. Incontrast, various cellular protective genes including transcription factors, Nrf2regulated antioxidants, and phase II responsive genes are down-regulated by smoke exposure (Halappanavar et al., 2009; Rangasamy et al., 2009). Genes involved in xenobiotic metabolic processes including Ahrr, Cyp1a1, and Cyp1b1 are up-regulated on day 4 after injection of hCB-MSCs. This finding can be explained by AHRR repression of Cyp1a1 through binding to its xenobiotic response element sequence. The expression of Cyp1b1 was transiently increased (up-regulated on day 4 and down-regulated on day 14 after injection of hCB-MSCs). $H b b$ and $H b a$, which were highly up-regulated on days 1 and 14 after injection of hCB-MSCs, are involved in oxygen transport from the lung to various peripheral tissues and are expressed in alveolar cells to perform antioxidant functions (Ahmad and Mahmood, 2016; Grek et al., 2011; Huffman et al., 2000; Macari and Lowrey, 2011; Newton et al., 2006).

Serpina is an inhibitor of serine proteases with proteolytic activity, particularly against elastase (Benarafa et al., 2007). The elastase versus anti-elastase hypothesis is one of the most important mechanistic theories for the development of smokinginduced emphysema (Shapiro, 1995; Stone, 1983). An imbalance between elastase and anti-elastase resulting from overexpression of elastase compared with anti-elastase causes alveolar destruction and airspace enlargement. Macrophageand neutrophil-derived elastase-deficient mice are significantly protected from the development of emphysema compared to wild-type mice (Hautamaki et al., 1997; Shapiro et al., 2003).

Therefore, MSCs may have regenerative roles that reestablish a balance between elastase and anti-elastase by upregulating Serpina expression. Egr1-deficient mice and lung fibroblasts exhibit lower metalloproteinase activity after inflammatory stimuli (Banks et al., 2005). This previous report suggests that EGR-1,by regulating metalloproteinases, is important in the development of COPD. We found that Egr1 was downregulated following injection of hCB-MSCs at all time points analyzed and that this alteration may inhibit metalloproteinase activity. Collectively, according to the cigarette smoke emphysema model, MSC sup-regulate anti-oxidant functions and down-regulate proteinase activity.

There were some limitations to this study. One limitation is that the differential change in gene expression profiles did not involve exposure to normal air and exposure to normal air plus hCB-MSCs. Therefore, further studies of mice exposed to normal air and the use of injected cell controls are needed to correctly identify the detailed regeneration mechanisms of stem cells. Another limitation is that our microarray experiment used whole lungs. Although we determined organ-level changes in molecular signals following the injection of hCB-MSCs, it remains unclear how the gene profiles change in individual cells in the lung such as bronchial and alveolar epithelial cells, fibroblasts, and endothelial cells. More advanced techniques are needed to analyze the gene expression profiles using sections of the lung tissues or specific lung cell types.

This is the first study to determine the time-dependent molecular changes induced by treatment with hCB-MSCs in a cigarette smoke-induced COPD model. MSCs systemically injected into an animal disappear within several days; it is thought that these cells rarely differentiate into tissue resident cells in the lungs, such as epithelial cells. Therefore, studies focused on molecular changes in host tissues may reveal the regenerative mechanisms of MSCs. Our present report improves the understanding of the regenerative mechanisms induced by injection of hCB-MSCs based on the gene expression profile changes in a cigarette smoke-induced COPD animal model.

Note: Supplementary information is available on the Molecules and Cells website (www.molcells.org).

\section{ACKNOWLEDGMENTS}

The authors thank the members of the Asan Medical Center animal facility for their technical expertise. This study was supported by a grant from the Korean Health Technology R\&D Project, Ministry of Health \& Welfare, Republic of Korea (no. $\mathrm{HI} 14 \mathrm{C} 1487)$ and research funds from the National Research Foundation of Korea (NRF-2015K1A4A3046807).

\section{REFERENCES}

Aaron, S.D., Vandemheen, K.L., Fergusson, D., Maltais, F., Bourbeau, J., Goldstein, R., Balter, M., O'Donnell, D., Mclvor, A., Sharma, S., et al. (2007). Tiotropium in combination with placebo salmeterol, or fluticasone-salmeterol for treatment of chronic obstructive pulmonary disease: a randomized trial. Ann. Intern. Med. 146, 545-555.

Ahmad, M.K., and Mahmood, R. (2016). Protective effect of taurine against potassium bromate-induced hemoglobin oxidation, oxidative stress, and impairment of antioxidant defense system in blood. Environ. Toxicol. 31, 304-313.

Banks, M.F., Gerasimovskaya, E.V., Tucker, D.A., Frid, M.G., Carpenter, T.C., and Stenmark, K.R. (2005). Egr-1 antisense oligonucleotides inhibit hypoxia-induced proliferation of pulmonary artery adventitial fibroblasts. J. Appl. Physiol. (1985) 98, 732-738.

Benarafa, C., Priebe, G.P., and Remold-O'Donnell, E. (2007). The neutrophil serine protease inhibitor serpinb1 preserves lung defense functions in Pseudomonas aeruginosa infection. J. Exp. Med. 204, 1901-1909.

Calverley, P.M., Anderson, J.A., Celli, B., Ferguson, G.T., Jenkins, C., Jones, P.W., Yates, J.C., and Vestbo, J. (2007). Salmeterol and fluticasone propionate and survival in chronic obstructive pulmonary disease. N. Engl. J. Med. 356, 775-789.

Chen, Z.H., Kim, H.P., Ryter, S.W., and Choi, A.M. (2008). Identifying targets for COPD treatment through gene expression analyses. Int. J. Chron. Obstruct. Pulmon. Dis. 3, 359-370.

Grek, C.L., Newton, D.A., Spyropoulos, D.D., and Baatz, J.E. (2011). Hypoxia up-regulates expression of hemoglobin in alveolar epithelial cells. Am. J. Respir. Cell Mol. Biol. 44, 439-447.

Halappanavar, S., Russell, M., Stampfli, M.R., Williams, A., and Yauk, C.L. (2009). Induction of the interleukin 6/ signal transducer and activator of transcription pathway in the lungs of mice sub-chronically exposed to mainstream tobacco smoke. BMC Med. Genomics 2, 56.

Hautamaki, R.D., Kobayashi, D.K., Senior, R.M., and Shapiro, S.D. (1997). Requirement for macrophage elastase for cigarette smoke-induced emphysema in mice. Science 277, 2002-2004.

Henning, R.J., Sanberg, P., and Jimenez, E. (2014). Human cord blood stem cell paracrine factors activate the survival protein kinase Akt and inhibit death protein kinases JNK and p38 in injured cardiomyocytes. Cytotherapy 16, 1158-1168.

Huffman, L.J., Miles, P.R., Shi, X., and Bowman, L. (2000). Hemoglobin potentiates the production of reactive oxygen species by alveolar macrophages. Exp. Lung Res. 26, 203-217.

Huh, J.W., Kim, S.Y., Lee, J.H., Lee, J.S., Van Ta, Q., Kim, M., Oh, Y.M., Lee, Y.S., and Lee, S.D. (2011). Bone marrow cells repair cigarette smoke-induced emphysema in rats. Am. J. Physiol. Lung Cell Mol. Physiol. 301, L255-266.

Katsha, A.M., Ohkouchi, S., Xin, H., Kanehira, M., Sun, R., Nukiwa, T., and Saijo, Y. (2011). Paracrine factors of multipotent stromal cells ameliorate lung injury in an elastase-induced emphysema model. Mol. Ther. 19, 196-203. 
Kim, Y.S., Kim, J.Y., Shin, D.M., Huh, J.W., Lee, S.W., and Oh, Y.M. (2014). Tracking intravenous adipose-derived mesenchymal stem cells in a model of elastase-induced emphysema. Tuberc. Respir. Dis. (Seoul) 77, 116-123.

Lee, R.H., Pulin, A.A., Seo, M.J., Kota, D.J., Ylostalo, J., Larson, B.L., Semprun-Prieto, L., Delafontaine, P., and Prockop, D.J. (2009). Intravenous hMSCs improve myocardial infarction in mice because cells embolized in lung are activated to secrete the anti-inflammatory protein TSG-6. Cell Stem Cell 5, 54-63.

Liang, X., Ding, Y., Zhang, Y., Tse, H.F., and Lian, Q. (2014). Paracrine mechanisms of mesenchymal stem cell-based therapy: current status and perspectives. Cell Transplant. 23, 1045-1059.

Macari, E.R., and Lowrey, C.H. (2011). Induction of human fetal hemoglobin via the NRF2 antioxidant response signaling pathway. Blood 117, 5987-5997.

MacNee, W., and Tuder, R.M. (2009). New paradigms in the pathogenesis of chronic obstructive pulmonary disease I. Proc. Am. Thorac. Soc. 6, 527-531.

Murphy, M.B., Moncivais, K., and Caplan, A.I. (2013). Mesenchymal stem cells: environmentally responsive therapeutics for regenerative medicine. Exp. Mol. Med. 45, e54.

Newton, D.A., Rao, K.M., Dluhy, R.A., and Baatz, J.E. (2006). Hemoglobin is expressed by alveolar epithelial cells. J. Biol. Chem. 281, 5668-5676.

Rabe, K.F., Hurd, S., Anzueto, A., Barnes, P.J., Buist, S.A., Calverley, P., Fukuchi, Y., Jenkins, C., Rodriguez-Roisin, R., van Weel, C., et al. (2007). Global strategy for the diagnosis, management, and prevention of chronic obstructive pulmonary disease: GOLD executive summary. Am. J. Respir. Crit. Care Med. 176, 532-555.

Rangasamy, T., Misra, V., Zhen, L., Tankersley, C.G., Tuder, R.M., and Biswal, S. (2009). Cigarette smoke-induced emphysema in $\mathrm{A} / \mathrm{J}$ mice is associated with pulmonary oxidative stress, apoptosis of lung cells, and global alterations in gene expression. Am. J. Physiol. Lung Cell Mol. Physiol. 296, L888-900.

Schweitzer, K.S., Johnstone, B.H., Garrison, J., Rush, N.I., Cooper, S., Traktuev, D.O., Feng, D., Adamowicz, J.J., Van Demark, M., Fisher, A.J., et al. (2011). Adipose stem cell treatment in mice attenuates lung and systemic injury induced by cigarette smoking. Am. J. Respir. Crit. Care Med. 183, 215-225.

Shapiro, S.D. (1995). The pathogenesis of emphysema: the elastase:antielastase hypothesis 30 years later. Proc. Assoc. Am Phys. 107, 346-352.

Shapiro, S.D., Goldstein, N.M., Houghton, A.M., Kobayashi, D.K. Kelley, D., and Belaaouaj, A. (2003). Neutrophil elastase contributes to cigarette smoke-induced emphysema in mice. Am. J. Pathol. 163, 2329-2335.

Stone, P.J. (1983). The elastase-antielastase hypothesis of the pathogenesis of emphysema. Clin. Chest Med. 4, 405-412.

Suga, H., Glotzbach, J.P., Sorkin, M., Longaker, M.T., and Gurtner, G.C. (2014). Paracrine mechanism of angiogenesis in adiposederived stem cell transplantation. Ann. Plast. Surg. 72, 234-241.

Yang, J.Y., Jin, J., Zhang, Z., Zhang, L., and Shen, C. (2013). Integration microarray and regulation datasets for chronic obstructive pulmonary disease. Eur. Rev. Med. Pharmacol. Sci. 17, 1923-1931.

Yoshida, T., and Tuder, R.M. (2007). Pathobiology of cigarette smoke-induced chronic obstructive pulmonary disease. Physiol. Rev. 87, 1047-1082.

Zandi-Nejad, K., Takakura, A., Jurewicz, M., Chandraker, A.K., Offermanns, S., Mount, D., and Abdi, R. (2013). The role of HCA2 (GPR109A) in regulating macrophage function. FASEB J. $27,4366-4374$ 